\title{
The value of clinical examination in diagnosing pelvic fractures in blunt trauma patients: a brief review
}

\author{
T. A. W. den Boer $\cdot$ M. Geurts $\cdot$ L. T. van Hulsteijn • \\ A. Mubarak $\cdot$ J. Slingerland $\cdot$ B. Zwart $\cdot$ \\ G. J. M. G. van der Heijden • T. J. Blokhuis
}

Received: 7 September 2010/ Accepted: 30 December 2010/Published online: 26 January 2011

(C) The Author(s) 2011. This article is published with open access at Springerlink.com

\begin{abstract}
Purpose of the study To evaluate the value of a pelvic $\mathrm{X}$-ray compared to clinical examination in diagnosing pelvic ring fractures, using computed tomography (CT) as the gold standard, in alert [Glasgow Coma Scale $(\mathrm{GCS}) \geq 13$ ] adult blunt trauma patients in the emergency room.

Methods A systematic literature search was performed in PubMed and Embase. The results were screened on their titles and abstracts using in- and exclusion criteria. Subsequently, the selected articles were critically appraised for their relevance and validity.

Results Two studies investigating the diagnostic value of clinical examination and pelvic X-ray compared to CT were identified. Both studies demonstrate higher negative predictive values for clinical examination $[0.99(95 \%$ confidence interval [CI] $0.98-1.0)$ and 1.0 (95\% CI 0.99-1.0)] compared to the negative predictive values of pelvic X-ray [0.98 (95\% CI 0.93-0.99) and 0.99 (95\% CI 0.99-1.0)]. The positive predictive values for clinical examination were low $[0.18$ (95\% CI $0.16-0.23)$ and 0.35 (95\% CI 0.30-0.42)] compared to pelvic X-ray [0.97 (95\% CI 0.96-0.98) and 0.97 (95\% CI 0.90-0.99)].
\end{abstract}

T. A. W. den Boer - M. Geurts · L. T. van Hulsteijn · A. Mubarak · J. Slingerland - B. Zwart · T. J. Blokhuis (ه) Department of Surgery, University Medical Center Utrecht (G04.228), P.O. Box 85500, 3508 GA Utrecht, The Netherlands e-mail: T.J.Blokhuis@umcutrecht.nl

T. A. W. den Boer - M. Geurts · L. T. van Hulsteijn ·

A. Mubarak · J. Slingerland - B. Zwart .

G. J. M. G. van der Heijden

Julius Center for Health Sciences and Primary Care,

University Medical Center Utrecht, Utrecht, The Netherlands
Conclusions In alert blunt trauma patients, pelvic X-ray only has additional diagnostic value for the detection of pelvic ring fractures if the clinical examination is positive. Pelvic X-ray should not be performed if the clinical examination is negative. In this manner, the expenditure of time, costs, and radiation are optimized.

Keywords Pelvic fractures - Clinical examination . Pelvic X-ray

\section{Introduction}

Pelvic fractures are frequently the result of high-energy blunt trauma and are associated with significant morbidity and mortality, most commonly due to hemorrhage [1]. Therefore, according to Advanced Trauma Life Support $\left(\mathrm{ATLS}^{\circledR}\right)$ guidelines, screening series of radiographs performed in all trauma patients must include a pelvic X-ray, in order to timely identify patients at risk of hemorrhage [2]. Radiographs are associated, however, with additional costs and harmful radiation. Also, in a busy emergency department with limited personnel or other resources, valuable time is invested in obtaining radiographs. Although the radiograph itself only takes seconds, the positioning of a film under the patient and interruption of the team during the primary survey make it a procedure that takes at least more than several minutes. Moreover, clinical examination of the pelvis is routinely performed in the work-up of trauma patients. Clinical examination consists of the establishment of pelvic stability. Manual compression of the iliac crests is performed to assess abnormal movement or bony pain. If the pelvis seems to be stable, cautious manual distraction of the iliac crests is performed, also evaluating for abnormal movement or 
bony pain. It takes very little time and, especially in alert patients, might suffice to detect pelvic fractures. If, based on both clinical examination and a pelvic X-ray, a pelvic fracture is suspected, almost always a computed tomography (CT) scan will follow to confirm the fracture and investigate its precise location and configuration.

If clinical examination alone would be sufficiently accurate for diagnosing pelvic fractures, this would reduce pelvic X-ray-associated radiation exposure, cut down costs for resource use, but, most importantly, save valuable time in the work-up after blunt trauma. It may also influence the availability of resources in the emergency department. For this report, the literature was systematically reviewed to investigate whether a pelvic radiograph has additional value compared to clinical examination alone in diagnosing pelvic fractures in alert [Glasgow Coma Scale (GCS) $\geq$ 13] patients after blunt trauma.

\section{Materials and methods}

\section{Search strategy and selection}

On 12 May 2010, a search was performed in MEDLINE and EMBASE for studies which included (synonyms of) the determinants, clinical examination and pelvic X-ray, and outcome of pelvic fracture (Table 1). A comprehensive search strategy was performed to retrieve relevant records. The inclusion and exclusion criteria were defined before the search was conducted: studies not comparing clinical examination to pelvic X-ray or not using CT as the external diagnostic criterion were excluded. The title and abstract of each record retrieved was independently screened by two authors. Inclusion of records was based on the full consensus of the two authors. Initial disagreement was resolved during discussion, but when doubt remained, the full-text publication was obtained and a third author decided.

\section{Critical appraisal}

All selected papers were independently critically appraised by two authors for their relevance and quality of methods. The appraisal criteria used are shown in Table 2.

Appraisal results are based on the full consensus of two authors. Initial appraisal disagreement was resolved during discussion, but when doubt remained, a third author decided.

\section{Data analysis}

To compare the results of the selected studies, positive predictive values (PPV), negative predictive values (NPV), positive likelihood ratios (PLR), and negative likelihood ratios (NLR) were (re-)calculated. Positive and negative predictive values describe the percentage of correct prediction in cases with a positive or negative result, respectively. In this study, this describes the presence or absence of pelvic fractures in case of a positive or negative clinical examination. The likelihood ratio describes how much the odds of a pelvic fracture increase when the clinical examination is positive or decrease when the clinical examination is negative.

Table 1 Search strategy and terms

\begin{tabular}{|c|c|c|}
\hline Database & Search & Hits \\
\hline $\begin{array}{l}\text { EMBASE } \\
12-5-2010\end{array}$ & $\begin{array}{l}\text { (Fracture:ti,ab OR fractures:ti,ab OR fractured:ti,ab OR disruption:ti,ab OR disruptions:ti,ab OR dysruption:ti,ab OR } \\
\text { dysruptions:ti,ab OR displacement:ti,ab OR displacements:ti,ab OR injury:ti,ab OR injuries:ti,ab OR trauma:ti,ab OR } \\
\text { traumas:ti,ab OR rupture:ti,ab OR ruptures:ti,ab) AND (pelvis:ti,ab OR pelvic:ti,ab OR 'open book':ti,ab OR ‘vertical } \\
\text { shear':ti,ab) AND (clinical:ti,ab OR physical:ti,ab OR pelvis:ti,ab OR pelvic:ti,ab) AND (examination:ti,ab OR } \\
\text { examinations:ti,ab OR sign:ti,ab OR signs:ti,ab OR impression:ti,ab OR impressions:ti,ab OR finding:ti,ab OR } \\
\text { findings:ti,ab OR examined:ti,ab OR assessment:ti,ab OR assessments:ti,ab OR presentation:ti,ab OR presentations:ti,ab } \\
\text { OR test:ti,ab OR tests:ti,ab OR testing:ti,ab) AND ('x ray’:ti,ab OR 'x rays':ti,ab OR 'plain film' :ti,ab OR 'plain } \\
\text { films':ti,ab OR roentgenogram:ti,ab OR roentgenograms:ti,ab OR imaging:ti,ab OR radiologic:ti,ab OR } \\
\text { radiological:ti,ab OR radiologically:ti,ab OR radiograph:ti,ab OR radiographs:ti,ab OR radiography:ti,ab OR } \\
\text { radiographic:ti,ab OR radiographics:ti,ab OR radiographically:ti,ab) }\end{array}$ & 1,310 \\
\hline $\begin{array}{l}\text { MEDLINE } \\
12-5-2010\end{array}$ & $\begin{array}{l}\text { (Fracture[TIAB] OR fractures[TIAB] OR fractured[TIAB] OR disruption[TIAB] OR disruptions[TIAB] OR } \\
\text { dysruption[TIAB] OR dysruptions[TIAB] OR displacement[TIAB] OR displacements[TIAB] OR injury[TIAB] OR } \\
\text { injuries[TIAB] OR trauma[TIAB] OR traumas[TIAB] OR rupture[TIAB] OR ruptures[TIAB]) AND (pelvis[TIAB] OR } \\
\text { pelvic[TIAB] OR open book[TIAB] OR vertical shear[TIAB]) AND (clinical [TIAB] OR physical[TIAB] OR } \\
\text { pelvis[TIAB] OR pelvic[TIAB]) AND (examination[TIAB] OR examinations[TIAB] OR sign[TIAB] OR signs[TIAB] } \\
\text { OR impression[TIAB] OR impressions[TIAB] OR finding[TIAB] OR findings[TIAB] OR examined[TIAB] OR } \\
\text { assessment[TIAB] OR assessments[TIAB] OR presentation [TIAB] OR presentations[TIAB] OR test[TIAB] OR } \\
\text { tests[TIAB] OR testing[TIAB]) AND (x ray[TIAB] OR x rays[TIAB] OR plain film [TIAB] OR plain films[TIAB] OR } \\
\text { roentgenogram[TIAB] OR roentgenograms[TIAB] OR imaging[TIAB] OR radiologic[TIAB] OR radiological [TIAB] } \\
\text { OR radiologically[TIAB] OR radiograph [TIAB] OR radiographs[TIAB] OR radiography [TIAB] OR radiographic } \\
\text { [TIAB] OR radiographics [TIAB] OR radiographically[TIAB]) }\end{array}$ & 1,108 \\
\hline
\end{tabular}


Table 2 Critical appraisal

\begin{tabular}{|c|c|c|c|c|c|}
\hline \multirow{2}{*}{$\begin{array}{l}\text { Study } \\
\text { (number of patients) }\end{array}$} & \multicolumn{3}{|c|}{ Relevance } & \multicolumn{2}{|c|}{ Quality of methods } \\
\hline & Domain & Determinant & Outcome & Blinding & $\begin{array}{l}\text { Standardization } \\
\text { assessments }\end{array}$ \\
\hline Duane et al. $2008(n=1,388)$ & $o$ & $\bullet$ & $\bullet$ & $o$ & $o$ \\
\hline Gonzalez et al. $2002(n=2,167)$ & $o$ & - & $o$ & - & - \\
\hline
\end{tabular}

- Adequate

$o$ Inadequate, doubtful

Relevance appraisal criteria:

Domain:

- Adults and Glasgow Coma Scale (GCS) $\geq 13$

$o$ Adults or GCS $\geq 13$

Determinant:

- Both clinical examination and pelvic X-ray are performed in all patients

$o$ Clinical examination and pelvic X-ray are not performed in all patients

Outcome:

- Pelvic fracture confirmed by CT in all patients

$o$ Pelvic fracture confirmed by CT in not all patients

Quality of methods appraisal criteria:

Standardization of assessments:

- All examinations were performed according to a pre-specified protocol

$o$ Not all examinations were performed according to a pre-specified protocol

Blinding:

- Clinical examination, pelvic X-ray, and CT were independently reviewed

$o$ Clinical examination, pelvic X-ray, and CT were not independently reviewed

\section{Results}

The search resulted in 1,316 unique records. After appraisal for relevance, only two investigations remained for critical appraisal for the quality of the methods used.

Duane et al. [3] prospectively compared clinical examination to plain radiographs, and both modalities to CT scanning in patients older than 16 years with blunt trauma. In total, 1,388 patients were included. The results of a separate analysis for the diagnostic accuracy of clinical examination was performed for the 1,059 patients presenting with a GCS $>13$. A total of 168 pelvic fractures were identified by CT, of which 101 were present in the cohort of patients with a GCS $>13$, corresponding with a pre-test probability of 0.12 [95\% confidence interval (CI) $0.10-0.14]$ for the total cohort and $0.10 \quad(95 \% \mathrm{CI}$ $0.08-0.12$ ) for those with GCS $>13$. In the cohort of patients with a GCS $>13$, clinical examination was associated with a PPV of 0.18 (95\% CI 0.16-0.23) and an NPV of 0.99 (95\% CI 0.98-1.0). The likelihood ratio for the positive clinical examination was 1.94 and for the negative examination, this was 0.07 . For pelvic X-ray, a PPV and NPV of, respectively, 0.98 (95\% CI 0.93-0.99) and 0.97 (95\% CI 0.96-0.98) was reported for the total cohort; a separate analysis for those with a GCS $>13$ was not reported. The likelihood ratio for the positive and negative pelvic X-ray were 322 (95\% CI 104-999) and 0.21 (95\% CI $0.15-0.28)$, respectively. All fractures identified by pelvic X-ray were identified by clinical examination as well (sensitivity $100 \%$ ). Because of the lack of a separate analysis for pelvic X-ray in patients with GCS $>13$, it was not possible to calculate the added value of pelvic X-ray compared to clinical examination solely in this report.

Gonzalez et al. [4] evaluated 2,176 consecutive patients with a GCS $>13$ after a blunt trauma. Clinical examination and pelvic radiographs were performed in all patients, followed by CT if either clinical examination (255 patients) or pelvic radiograph (77 patients) was positive, or if during admission or after discharge symptoms of pelvic fractures appeared (no patients). Although not all of the test characteristics were presented, it was possible to calculate missing data from the text. Ninety-seven pelvic fractures were identified by CT (pre-test probability $0.05,95 \% \mathrm{CI}$ 0.04-0.05). Their results demonstrated a PPV of $0.35(95 \%$ CI 0.30-0.42) and an NPV of 1.0 (95\% CI 0.99-1.0) for clinical examination, while the likelihood ratio for the positive and negative clinical examination were 11.7 (95\% CI 10-14) and 0.08 (95\% CI 0.04-0.16), respectively. Regarding pelvic X-ray, a PPV and NPV of 0.97 (95\% CI 0.90-0.99) and 0.99 (95\% CI 0.99-1.0) were found, 
whereas the likelihood ratio for the positive and negative pelvic X-ray were 600 (95\% CI 193-1,864) and 0.13 (95\% CI $0.08-0.22$ ), respectively. The added value of pelvic $\mathrm{X}$-ray for ruling in patients with a pelvic fracture was, compared to clinical examination alone, 0.62. For ruling out patients with pelvic fractures, the added value of pelvic $\mathrm{X}$-ray was 0.

\section{Discussion}

ATLS ${ }^{\circledR}$ guidelines recommend routine performance of pelvic radiographs in blunt trauma patients, despite several drawbacks associated with obtaining them. In the present report, the literature was reviewed in a systematic manner to find out whether this is justified.

A literature search and subsequent screening using strict criteria resulted in two studies, both demonstrating higher negative predictive values for clinical examination (Table 3), suggesting that pelvic radiographs have no additional value in ruling out pelvic fractures when the clinical examination is negative in alert trauma patients after blunt trauma. By contrast, when the clinical examination cannot rule out a pelvic fracture, a pelvic radiograph should be ordered to accurately determine the pelvic fracture status of the patient.

The following considerations should be taken into account when interpreting these data. In the study by Duane et al. [3], several methodological flaws were detected. In the study by Gonzalez et al. [4], there was considerable work-up bias, i.e., the CT scan as the external reference standard was not performed in a substantial number of patients. Both studies investigated patients with a GCS $>13$. The purpose of this report was to investigate all alert patients, defined as a GCS $\geq 13$. Due to the small number of studies and the reported methodological limitations, the evidence for the diagnostic accuracy of a pelvic radiograph in blunt trauma patients in addition to clinical examination is considered to be rather weak.

However, applying a stepwise triage approach for pelvic fractures in patients after blunt trauma in daily practice has several benefits. First, it will save time in emergency situations because a pelvic radiograph can be omitted in a considerable number of patients. In busy emergency departments, or in a triage scenario where multiple patients have to be evaluated simultaneously, resources are sometimes limited and any redundant step in the work-up should be avoided. Also, by adopting the proposed strategy, many patients will not be exposed unnecessarily to radiation. Although the radiation exposure per patient is low, the large number of patients makes this consideration important. The avoidance of radiographs will also save money, as demonstrated in the study by Duane et al. [3]. On the other hand, there are some disadvantages. Changing a protocol requires good communication in the trauma team, as radiographs will only be needed in a select number of patients. In addition, despite the high negative predictive values, a few pelvic fractures were missed by clinical examination in both studies. These missed fractures eventually had no therapeutic consequences, and, so, turned out

Table 3 Results

\begin{tabular}{|c|c|c|}
\hline Author of study (year of publication) & Duane et al. (2008) & Gonzalez et al. (2002) \\
\hline \multirow[t]{2}{*}{ Pre-test probability $(95 \% \mathrm{CI})^{\mathrm{a}}$} & $0.10(0.08-0.12)^{*}$ & $0.05(0.04-0.05)$ \\
\hline & $0.12(0.10-0.14)^{* *}$ & \\
\hline \multicolumn{3}{|l|}{ Clinical examination } \\
\hline Positive predictive value $(95 \% \mathrm{CI})$ & $0.18(0.16-0.23)^{*}$ & $0.35(0.30-0.42)$ \\
\hline Negative predictive value $(95 \% \mathrm{CI})$ & $0.99(0.98-1.0)^{*}$ & $1.0(0.99-1.0)$ \\
\hline \multicolumn{3}{|l|}{ Pelvic X-ray } \\
\hline Positive predictive value $(95 \% \mathrm{CI})$ & $0.98(0.93-0.99)^{* *}$ & $0.97(0.90-0.99)$ \\
\hline Added value of pelvic X-ray for ruling in pelvic fractures ${ }^{b}$ & Not available & 0.62 \\
\hline Negative predictive value $(95 \% \mathrm{CI})$ & $0.97(0.96-0.98)^{* *}$ & $0.99(0.99-1.0)$ \\
\hline Added value of pelvic X-ray for ruling out pelvic fractures ${ }^{b}$ & Not available & 0.0 \\
\hline \multicolumn{3}{|l|}{ Remaining uncertainty after best test for: } \\
\hline Ruling in & 0.02 & 0.03 \\
\hline Ruling out & 0.01 & 0.0 \\
\hline
\end{tabular}

CI confidence interval

* In patients with a Glasgow Coma Scale $\geq 13$

** In all patients

${ }^{\text {a }}$ Pre-test probability = prevalence of pelvic fractures on CT scan

${ }^{\mathrm{b}}$ Difference in PPV and NPV between clinical examination alone and pelvic X-ray as well as clinical examination 


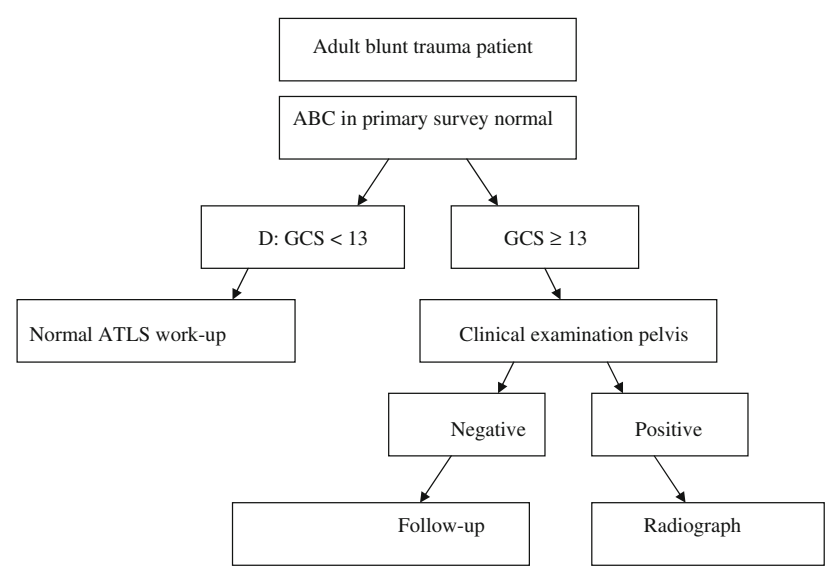

Fig. 1 Flowchart for the work-up of the pelvis in alert trauma patients

to be clinically insignificant. A proposed flowchart for the work-up of the group of patients under consideration in this review is shown in Fig. 1.

Although only two articles answered the clinical question specifically, several studies investigated the diagnostic value of clinical examination using pelvic radiographs as a reference test (and not the gold standard CT). In these studies [5-14], negative predictive values ranging from 0.58 to 1.0 were found, with six out of these ten studies demonstrating a negative predictive value of 1.0. In other words, if clinical examination is negative, pelvic fractures on radiographs will be highly unlikely, and, thus, pelvic $\mathrm{X}$-ray will have no additional value. These results support the conclusion that, in the case of a negative clinical examination, a pelvic radiograph can be omitted. In case of positive examination, radiographs do have additional value in showing some false-positive clinical examination results, and, hence, can best be used as a rule-out strategy in pelvic fracture triage.

In conclusion, in alert patients following blunt trauma, negative findings on clinical examination justify the omission of standard pelvic radiographs. In this manner, the work-up of trauma patients and the application of resources can be optimized.

Conflict of interest None.
Open Access This article is distributed under the terms of the Creative Commons Attribution Noncommercial License which permits any noncommercial use, distribution, and reproduction in any medium, provided the original author(s) and source are credited.

\section{References}

1. Grotz MR, Allami MK, Harwood P, Pape HC, Krettek C, Giannoudis PV. Open pelvic fractures: epidemiology, current concepts of management and outcome. Injury. 2005;36:1-13.

2. Young JW, Burgess AR, Brumback RJ, Poka A. Pelvic fractures: value of plain radiography in early assessment and management. Radiology. 1986;160:445-51.

3. Duane TM, Dechert T, Wolfe LG, Brown H, Aboutanos MB, Malhotra AK, Ivatury RR. Clinical examination is superior to plain films to diagnose pelvic fractures compared to CT. Am Surg. 2008;74:476-9.

4. Gonzalez RP, Fried PQ, Bukhalo M. The utility of clinical examination in screening for pelvic fractures in blunt trauma. J Am Coll Surg. 2002;194:121-5.

5. Civil ID, Ross SE, Botehlo G, Schwab CW. Routine pelvic radiography in severe blunt trauma: is it necessary? Ann Emerg Med. 1988;17:488-90.

6. Duane TM, Cole FJ Jr, Weireter LJ Jr, Britt LD. Blunt trauma and the role of routine pelvic radiographs. Am Surg. 2001;67:849-52.

7. Duane TM, Tan BB, Golay D, Cole FJ Jr, Weireter LJ Jr, Britt LD. Blunt trauma and the role of routine pelvic radiographs: a prospective analysis. J Trauma. 2002;53:463-8.

8. Ersoy G, Karcioğlu O, Enginbaş Y, User N. Should all patients with blunt trauma undergo 'routine' pelvic X-ray? Eur J Emerg Med. 1995;2:65-8.

9. Gross EA, Niedens BA. Validation of a decision instrument to limit pelvic radiography in blunt trauma. J Emerg Med. 2005;28:263-6.

10. McCormick JP, Morgan SJ, Smith WR. Clinical effectiveness of the physical examination in diagnosis of posterior pelvic ring injuries. J Orthop Trauma. 2003;17:257-61.

11. Salvino CK, Esposito TJ, Smith D, Dries D, Marshall W, Flisak M, Gamelli RL. Routine pelvic X-ray studies in awake blunt trauma patients: a sensible policy? J Trauma. 1992;33:413-6.

12. Shlamovitz GZ, Mower WR, Bergman J, Chuang KR, Crisp J, Hardy D, Sargent M, Shroff SD, Snyder E, Morgan MT. How (un)useful is the pelvic ring stability examination in diagnosing mechanically unstable pelvic fractures in blunt trauma patients? J Trauma. 2009;66:815-20.

13. Yugueros $P$, Sarmiento JM, Garcia AF, Ferrada R. Unnecessary use of pelvic X-ray in blunt trauma. J Trauma. 1995;39:722-5.

14. Ham SJ, van Walsum AD, Vierhout PA. Predictive value of the hip flexion test for fractures of the pelvis. Injury. 1996;27:543-4. 